\title{
A case of Degos disease in pregnancy
}

\author{
Sapna Sharma', Barbara Brennan', Ray Naden ${ }^{2}$ and \\ Patrick Whelan ${ }^{3,4}$
}

sagepub.co.uk/journalsPermissions.nav DOI: $10.1177 / 1753495 \times 16652006$ obm.sagepub.com

@SAGE

\begin{abstract}
Degos disease is characterized as a rare systemic vaso-occlusive disorder, although the exact pathophysiology is uncertain. Fewer than 200 patients have been reported in the literature, and only two reports describe the course of the disease during pregnancy. Here, we present the first reported case of the course of pregnancy in a woman with the systemic form of Degos disease. The patient had been diagnosed with Degos prior to pregnancy and was monitored throughout the duration of the pregnancy. Her presentation and treatment are described. There was no further exacerbation secondary to the pregnancy itself; the pregnancy course was uncomplicated and the baby unaffected to date.
\end{abstract}

\section{Keywords}

General medicine, high-risk pregnancy

Date received: 8 January 2016; accepted: 6 May 2016

\section{Case}

A 25-year-old nulliparous woman was seen in consultation in 2010 by a community-based Dermatologist for a new onset of skin lesions. The first lesions appeared on her wrist, and eventually progressed to include her arms, abdomen and legs. They were described as small, white, punctate lesions with an erythematous halo. Biopsy results were inconclusive for Degos, describing lymphocytic infiltrate with dermal mucinosis. The patient was then referred to a tertiary care center where her history was reviewed by multiple Dermatologists. The consensus amongst the group was that the presentation was consistent with Degos disease. In 2013, the patient had an episode of headache with aura, as well as slurred speech, and was assessed in the emergency department. Her neurologic exam, blood work and non-contrast computerized tomography scan of the brain were normal. However, given the history of Degos disease, she underwent a magnetic resonance imaging. This demonstrated multiple (approximately 25) tiny juxtacortical, subcortical and deep white matter Fluid-attenuated inversion recovery (FLAIR) foci scattered throughout the cerebral hemispheres. No acute ischemia was identified. The interpretation was that the findings were consistent with Degos disease. Upper and lower endoscopy and biopsies were performed and were negative. A consultation with the Thrombosis team was obtained, and the decision was made to initiate therapy with acetyl salicylic acid (ASA) $81 \mathrm{mg}$.

Laboratory investigations including coagulation function, lupus anticoagulant, anticardiolipin antibody, prothrombin time, partial thromboplastin time and international normalized ratio were all within normal limits. Anti-nuclear antibody titer was less than $1: 40$.

Her existing skin lesions were monitored throughout the pregnancy and were unchanged. Furthermore, she did not develop any other gastrointestinal (GI) or neurologic symptoms for the remainder of the pregnancy. There were no fetal concerns throughout the pregnancy.

The patient underwent a planned and uncomplicated Cesarean section at 38 weeks and one day gestational age for persistent breech presentation. A live male infant was delivered weighing $3229 \mathrm{~g}$ with APGARS of 9 at $1 \mathrm{~min}$ and 9 at $5 \mathrm{~min}$. The baby was inspected for skin lesions at birth by the attending Neonatologist and none were found. The placenta was visually assessed and found to be normal. Pathology was performed, which demonstrated a normal umbilical cord with an eccentric insertion. The weight was $461 \mathrm{~g}$ and normal in dimension. The fetal surface appeared normal in color and was well vascularized. The maternal surface appeared unremarkable. There was no evidence of thrombosis or vasculitis.
The patient was reviewed at six weeks postpartum and was doing well. There had been no change in her cutaneous lesions, and no development of any extra cutaneous symptoms.

\section{Discussion}

Köhlmeier first described Degos disease in $1941 .{ }^{1}$ Degos disease is characterized as a rare systemic vaso-occlusive disorder, although the exact pathophysiology is uncertain. Some have theorized that Degos results from a coagulopathy, vasculitis or endothelial cell dysfunction. ${ }^{2}$ However, anticoagulation strategies geared to a coagulopathy and immunosuppressive approaches successful with vasculitis have proven inadequate in treating systemic disease. ${ }^{2}$ Dermal capillaries and venules develop a thrombogenic microangiopathy, resulting in the characteristic raised papules with a red border and a depressed "china white" center. The majority of cases are limited to cutaneous lesions, although there can be evolution of the disease to include the gastrointestinal or nervous system. ${ }^{6-11}$ Until recently, the systemic form of the disease was almost universally fatal. ${ }^{12}$

To date, there are no clear guidelines with respect to treatment of Degos disease. There are reports that aspirin or dipyridamole has been effective. ${ }^{3,4}$ Heparin has been used with success in acutely ill patients, ${ }^{5,6}$ although other fibrinolytic agents have been ineffective. ${ }^{7}$ Immunosuppression with corticosteroids has been shown to worsen skin lesions and further complicate the course of the disease. ${ }^{8,9}$

The rarity of the condition is such that only two known cases of Degos disease in pregnancy exist in the literature. ${ }^{10,11}$ The first case reported in 1984 describes a patient with multiple characteristic skin lesions in the absence of any visceral, GI or neurologic involvement. One year after the onset of the disease she had a successful pregnancy. Her daughter had been followed for nine years and was found to have developed cutaneous lesions only. The second case of Degos disease in

'Department of Obstetrics and Gynecology, Division of Maternal Fetal Medicine, McMaster University, Hamilton, ON, Canada

${ }^{2}$ Department of Medicine, McMaster University, Hamilton, ON, Canada

${ }^{3}$ Division of Rheumatology, MassGeneral Hospital for Children and Harvard Medical School, Boston, MA, USA

${ }^{4}$ Heritage Provider Network, Los Angeles, CA, USA

\section{Corresponding author:}

Sapna Sharma, McMaster Children's Hospital, Rm HSC 4F, I200 Main St W, Hamilton, ON L8N 3Z5, Canada.

Email: ssharma2009@gmail.com 
pregnancy was reported in 1997. This patient also had cutaneous manifestations in the absence of any GI, visceral or neurologic abnormalities. The lesions appeared during her first pregnancy in 1992. Subsequent workup during her second pregnancy in 1997 demonstrated clinical and histopathological findings consistent with Degos disease. This patient's course was also characterized by cutaneous lesions without the development of visceral or neurological involvement. However, unlike the first case, this patient developed lesions in pregnancy, suggesting that pregnancy may have served as a precipitating event for the re-activation of the disease. In this case, in light of the positive anti phospholipid antibody (APA), oral anticoagulants or ASA were considered, but given the absence of previous thrombotic events or any GI or neurologic involvement, the decision was made not to treat. ${ }^{11}$

The present case, similar to the first one, offered the chance to monitor the disease throughout the duration of the pregnancy. This patient's cutaneous lesions remained unchanged throughout the pregnancy. She was managed solely on ASA $81 \mathrm{mg}$ daily with close surveillance by a multidisciplinary team. Previous case reports described patients with probable Degos disease with positive Anti nuclear antibody (ANA) and APA, although there are no reported cases of de novo development of positive antibodies. ${ }^{13}$ As previously mentioned, this patient was negative for ANA and APA early in pregnancy, and this remained unchanged in the third trimester. Furthermore, the course of her pregnancy was uncomplicated and resulted in the live birth of a healthy male infant at term. As one might predict from the good clinical outcome, this Degos patient lacked any of the chronic inflammatory or placental vaso-occlusive lesions seen in pre-term preeclampsia, or the ischemic-hypoxic change, decidual vasculopathy, chronic villitis or infarction evident in systemic lupus. ${ }^{14}$ This case demonstrates that the disease did not adversely affect the pregnancy in that the pregnancy course itself was uncomplicated and the baby was unaffected to date.

\section{Declaration of conflicting interests}

The author(s) declared no potential conflicts of interest with respect to the research, authorship, and/or publication of this article.

\section{Funding}

The author(s) received no financial support for the research, authorship, and/or publication of this article.

\section{Ethical approval}

Written informed consent for patient information was provided by the patient.

\section{Guarantor}

Dr Sapna Sharma.

\section{Contributorship}

All authors contributed equally to the drafting of the case report and have provided final approval of the version to be published.

\section{References}

1. Köhlmeier W. Multiple Hautnekrosen bei Thrombangitis obliterans. Arch Dermatol Syph (Wien) 1941; 181: 783-792.

2. Ball E, Newburger A and Ackerman AB. Degos' disease: a distinctive pattern of disease, chiefly of lupus erythematosus, and not a specific disease per se. Am J Dermatopathol 2003; 25: 308-320.

3. Drucker CR. Malignant atrophic papulosis: response to antiplatelet therapy. Dermatologica 1990; 180: 90-92.

4. Stahl D, Thomsen K and Hou-Jensen K. Malignant atrophic papulosis: treatment with aspirin and dipyridamole. Arch Dermatol 1978; 114: 1687-1689.

5. Rivoire MJ. Degos' malignant atrophic papulosis. Outcome after 28 months' treatment with anticoagulants. Bull Soc Fr Dermatol Syphiligr 1971; 78: 18-19.

6. Guven FO, Bozdag KE, Ermete M, et al. Degos' disease. Int $J$ Dermatol 2000; 39: 361-362.

7. Chave TA, Varma S, Patel GK, et al. Malignant atrophic papulosis (Degos' disease): clinicopathological correlations. J Eur Acad Dermatol Venereol 2001; 15: 43-45.

8. Ojeda Cuchillero RM, Sanchez Regana M and Umbert Millet P. Benign cutaneous Degos' disease. Clin Exp Dermatol 2003; 28: 145-147.

9. Powell J, Bordea C, Wojnarowska F, et al. Benign familial Degos disease worsening during immunosuppression. $\mathrm{Br} J$ Dermatol 1999; 141: 524-527.

10. Moulin G, Barrut D, Franc MP, et al. Familial Degos' atrophic papulosis (mother-daughter). Ann Dermatol Venereol 1984; 111: 149-155.

11. Bogenrieder T, Kuske M, Landthaler M, et al. Benign Degos' disease developing during pregnancy and followed for 10 years. Acta Derm Venereol 2002; 82: 284-287.

12. Shapiro LS, Toledo-Garcia AE and Farrell JF. Effective treatment of malignant atrophic papulosis (Kohlmeier-Degos disease) with treprostinil-early experience. Orphanet $J$ Rare Dis 2013; 8: 52.

13. High WA, Aranda J, Patel SB, et al. Is Degos' disease a clinical and histological end point rather than a specific disease? $\mathrm{J} \mathrm{Am}$ Acad Dermatol 2004; 50: 895-899.

14. Magid MS, Kaplan C, Sammaritano LR, et al. Placental pathology in systemic lupus erythematosus: a prospective study. Am J Obstet Gynecol 1998; 179: 226-234. 